\title{
Towards QoS-awareness of Context-aware Mobile Applications and Services
}

\author{
Katarzyna Wac \\ University of Geneva, CUI/OSG group, 24 rue General Dufour, 1211 Geneva 4, Switzerland \\ University of Twente, EWI/ASNA group, P.O.Box 217, 7500 AE Enschede, The Netherlands \\ Katarzyna.Wac@cui.unige.ch,k.e.wac@utwente.nl
}

\begin{abstract}
In our current connected wireless world, mobile devices are enabled to use various networking facilities. Although this enables mobile users to communicate any time and any place, it may also be very intrusive. There is a high need to manage the information stream a user receives on his/her mobile device. Context-awareness seems to be a promising way to manage this information stream and to provide the means to communicate at the right time in the right way.

Current context-aware applications benefit from the user context (e.g. location information), however, they do not consider the quality of service (QoS) offered by various networks (i.e. only best-effort QoS is considered). The research discussed in this paper focuses on a QoS- and context-aware service infrastructure supporting the development of mobile applications in a heterogeneous network environment. We argue that the use of context information helps to better capture the user's required QoS and improves the delivered QoS.
\end{abstract}

\section{Introduction}

The emergence of new wireless broadband networks and diverse miniaturized and personalized networked devices, give rise to variety of new mobile services in our daily life. Ultimately, these mobile services are executed as we move: in different places, at different times and under different conditions. Hence, these services get a continuously-changing information flow from their execution environment. The management of this flow becomes vital for mobile service delivery. As a result, the communication paradigm will need to shift from any time and any place into the right time in the right way, as the former may be very intrusive.

Context-awareness seems to be a promising way to manage this information, as context is any information that can be used to characterize the environment and situation of a user, (e.g. current location and time), and any object that is considered relevant to the interaction between the user and a mobile service [1].

For any mobile service, the underlying communication is provided by different networks; wireless and wired. Each network is responsible for a section of the 'endto-end' communication path between the mobile user and application server placed in 


\section{Katarzyna Wac}

a service provider network. Figure 1 presents the path decomposition. The mobile operator network comprises wireless access network and wired core network.

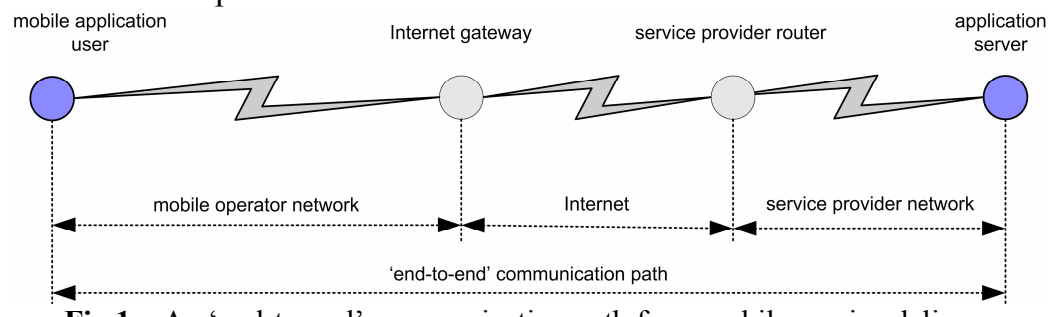

Fig.1. An 'end-to-end' communication path for a mobile service delivery

Mobile connectivity, i.e., persistency of a wireless connection during the act of being mobile, and the quality of service $\left(\mathrm{QoS}^{1}\right)$ offered by this connection, are the most critical factors for the success of mobile service delivery. However, current rules for a connectivity choice are rather simple - a default wireless connection is chosen at service-design time and assumptions are made regarding its offered QoS. Because a wireless link is a bottleneck in the end-to-end communication path, the assumptions regarding its offered QoS imply the assumption for the end-to-end offered QoS. Consequently, current mobile services are delivered with a best-effort (end-to-end) quality, and without really considering the mobile user's required QoS.

The complication rises from the fact that nowadays various connectivity possibilities coexist offering different QoS, and current mobile applications are unaware of that. The choice of the wireless network is in most cases based on network availability only, and not its offered QoS. Moreover, the mobile user's QoS requirements are not really considered or assumed to be static and attempted to be met with a best-effort service. As a complication we also see that the mobile application does not 'learn' from the end-to-end QoS experienced along the service delivery. This QoS information is not logged or used further for QoS predictions, neither for a user himself nor for the other mobile users.

With respect to all given complications, we research on a QoS- and context-aware service infrastructure that supports the development of mobile applications in a heterogeneous network environment. Hence we argue that the mobile service infrastructure should not only be context-aware, but also QoS-aware. That means it needs to be aware of the user's required QoS and QoS offered by various networks at the mobile user's current location and time. A wireless connection should be chosen with respect to what is required and what is offered. If necessary, service delivery should be adapted to what is offered (e.g. by means of changing the application protocol parameters). We argue that QoS- and context-awareness improves delivered QoS. Moreover, we postulate that context-awareness would help to better capture the actual user QoS requirements as they can change along with the context change. For example, in the mobile healthcare application domain, the non-emergency to emergency context change may mean a change of the corresponding QoS requirements (i.e. from 'loose' to 'strict').

${ }^{1}$ e.g. user-based QoS like: cost, security level, etc., and technology-based QoS like network performance (speed, accuracy and dependability [2]), etc. 
Furthermore, we argue that QoS-awareness needs to be taken even broader. Namely, the actually delivered end-to-end QoS should be continuously logged by the mobile service infrastructure, and, based on its history, further used for QoS prediction. That would lead to the proactive QoS-aware and context-aware mobile service infrastructure. Moreover, we argue that the existence of a QoS-context source is necessary. This source would carry the responsibility of accumulation of logs on delivered end-to-end QoS from different mobile service users, and provision of predictions of this QoS to mobile applications. An end-to-end QoS may be then predicted along the particular trajectory traversed in a particular timeframe. Hence, our vision is a development of a 'route navigator' (like a GIS-based system, but also for indoor usage) enriched with a QoS prediction for a particular user's trajectory.

The following section gives an overview of the existing research on the topic. This is followed by explanation of our research context (section 3) and established research trajectory within this context (section 4). The state of our thesis is then discussed in section 5. The conclusion in section 6 summarizes our thesis goals and provides a critical evaluation of the work.

\section{State-of-the-art}

There has already been a lot of research on QoS and context-awareness as separate topics, hence this section presents only work that aims to solve one or more of the complications mentioned in section 1.

Projects like Equanet [3] developed a modeling-based performance evaluation method of the end-to-end QoS delivered to a mobile user over heterogeneous communication infrastructures. However, this project only focuses on two specific mobile services VoIP and mobile web browsing. In contrary, our research does not put constraints on the type of services, we consider.

The CELLO project [4] concentrates on the location-based performance assessment of wireless communication infrastructures. Data regarding network performance is stored in a GIS system. However, as a performance indicator this project only considers signal strength measurements and not the end-to-end QoS, as we do. Moreover, the overall goal of the project is to enhance the mobile operator network; the data is not used for mobile users, as we propose in our research.

The publication of [5] provides a framework for network-aware applications and indicates that application-level monitoring is one of the methods for application to be network-aware. However, as the end-to-end performance parameter they only consider end-to-end bandwidth, and no other QoS parameters, as we propose.

Similarly, [6] provides an idea on network resource awareness at the application level. They indicate user context as necessary information for an application to adapt. However, they do focus on the access network and not on end-to-end resource availability, as we do.

Finally, [7] discloses mobile connectivity information as context information vital for mobile service delivery. However, they do not indicate end-to-end QoS, but they focus on a wireless access network. Moreover, the mobile connectivity-context source 


\section{Katarzyna Wac}

they indicate will be based on the history of connectivity of a single user, and will be used for a user himself to derive further context information. Hence, it will not be used for other users, as we indicate in our research.

Based on this short representation of the ongoing research, we define the innovative contribution of our thesis to the existing state of the art as the fact that we take the end-to-end QoS characteristics as context information and we introduce the notion of a QoS-context source, which will be fed by users and will be used for users.

\section{Research context}

The context of our thesis is provided by the Freeband AWARENESS Dutch national project [8]. This project focuses on the research of an infrastructure that supports the development of context-aware and pro-active services and applications. AWARENESS validates this infrastructure through prototyping with mobile healthcare applications.

AWARENESS defines a three-layered architecture. The bottom layer of the

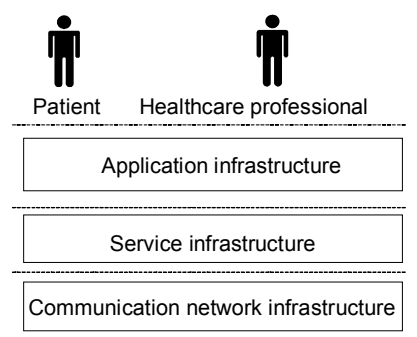

Fig.2. AWARENESS architecture architecture is the communication network infrastructure layer, offering seamless mobile connectivity (e.g. $2.5 \mathrm{G} / 3 \mathrm{G}$ and WLAN). This layer spans the 'end-to-end' communication path indicated in Figure 1.

The middle layer is the service infrastructure layer that provides an execution environment for mobile services. It provides generic functionality like service discovery and service context management function. The top layer is the application layer which offers generic application services like application-level context management and domain specific services like health tele-monitoring services, e.g., for the epilepsy, spasticity and chronic pain domains.

We position our research vertically across these three layers. We will incorporate the QoS requirements of service users (i.e., patients and healthcare professionals) and map them into system QoS requirements - into the requirements at the service infrastructure layer and then into the requirements at communication network infrastructure layer. To merge QoS-awareness and context-awareness, the context management function at the service infrastructure layer will be enriched with the management of QoS-context information. Moreover, we will develop a QoS-context source interacting with the service infrastructure layer. 


\section{Research trajectory}

Our research trajectory consists of a few consecutive phases: 1) analysis of researchrelated concepts (to identify the current situation and its problems) as a basis for formulation of our research questions, 2) putting forward a hypothesis on a possible solution and 3) defending the hypothesis, eventually proving it to be a valid theory. Figure 3 presents our research trajectory and particular actions taken in each of the phases.

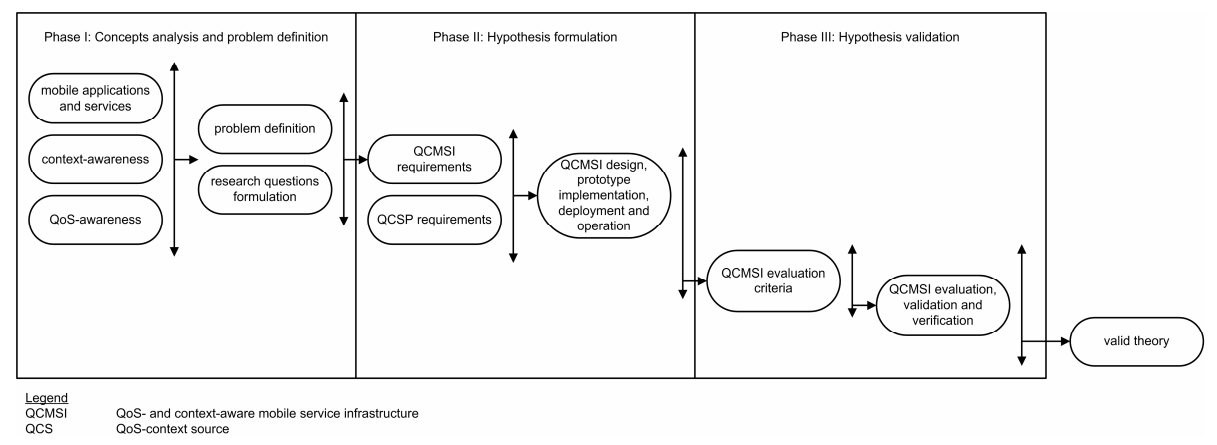

Fig.3. Research trajectory

Phase I In Phase I we start from the system related-concepts analysis and problem definition. We consider three main areas of literature review relevant for this research, namely a) mobile applications and services (e.g., current mobile healthcare applications), b) context-awareness (e.g., user/system context, current context-aware services and toolkits) and c) aspects of the QoS-awareness (e.g., what is QoS, QoS for a mobile services, QoS management). This study results in a problem analysis of current context-aware service infrastructures and their QoS-(un)awareness.

Furthermore, Phase I aims in deriving specific research questions. These questions will be based on understanding the existing service infrastructure and its problems.

Phase II Following the research questions, in Phase II we will derive a hypothesis of a possible solution for the identified problem. As we stated earlier, we research on a QoS- and context-aware service infrastructure that supports the development of mobile applications in a heterogeneous network environment. Due to the nature of the problem, we derive our hypothesis based on the repeatable service lifecycle model [9]. This model consists of the following phases: a) requirements (i.e., set of features service must conform; service user and system ${ }^{2}$ requirements), b) architectural design (describing the service organization in terms of structural elements, their composition, their interfaces and behavior), c) implementation (hardware, software and firmware used), d) deployment (service availability to users) and e) service operational phase (service maintenance).

Following this model, we will analyze requirements for the QoS- and context-aware service infrastructure and then requirements for QoS-context source. The QoS- and context-aware service infrastructure dictates the requirements for the QoS-context source.

\footnotetext{
${ }^{2}$ A system delivers a service; a service is an external observable behavior of the system.
} 
Based on the identified requirements, we will propose an architectural design for the QoS- and context-aware service infrastructure and its interface with QoS-context source.

Our hypothesis involves a prototype implementation and deployment of the system. Following the AWARENESS project goals, this will be done with mobile healthcare applications.

Phase III The system prototype will aim in proving such whether the proposed solution fits the identified earlier problem (Phase I) and conforms the identified requirements (Phase II). Hence in Phase III we will attempt to defend (or refute!) our hypothesis. Firstly we will derive evaluation criteria along which the prototyped system will be further validated and verified. The evaluation will be executed with real mobile healthcare service users and will aim to prove that use of context information indeed helps better capture the user's required QoS and improves the delivered QoS. Hence, we may for example derive a mean-opinion-score [10] for the electro-cardiogram (ECG) vital sign tele-monitoring service.

If we defend our hypothesis to be a theory, then we would like to indicate the utility of our solution for a mobile service in any application domain (e.g., mobile information retrieval, m-commerce, mobile gaming).

\section{Current work}

The research discussed in this paper started in November 2004 and consequently it is in a starting phase. Activities from Phase I - the problem definition and the highlights on the most important state-of-the-art research are presented in the first sections of this paper. In the remainder of this section we discuss consequently our research questions together with the initial ideas for Phase II.

\subsection{Research questions}

Research needs to be done on a context-aware mobile service infrastructure, to enrich it with QoS-awareness. Moreover, we need to define the services that a QoS-context source offers. Therefore, we define the following research questions:

- What are a mobile user's end-to-end QoS requirements? How to translate them into the system requirements?

- What end-to-end QoS context information is required at the context-aware service infrastructure level, how to get, it and how will it be used? How may context information improve end-to-end QoS actually delivered to a mobile user?

- What are the requirements for, and how to develop a QoS-context source? Where does this source get end-to-end QoS information from and to whom does it provide?

- Based on the data aggregated in a QoS-context source, what algorithms are needed to predict end-to-end QoS along the mobile user's trajectory traversed in a given timeframe? 


\subsection{Epilepsy tele-monitoring scenario}

The following future application scenario illustrates how an epileptic patient can benefit from a context- and QoS-aware mobile healthcare service infrastructure. This scenario we use further to derive service infrastructure requirements. We present the scenario in boxed paragraphs followed by explanations what technology we propose to support this scenario.

Sophie (28) is an epileptic patient living in the suburbs of Paris. Epilepsy is a neurological disorder, in which, from time to time, nerve cells of the brain release abnormal electrical impulses so-called seizures. This disorder affects Sophia's nervous system already for four years. Although the occurrence of a seizure is often sudden and unexpected, she does not feel limited in her daily active life. It is because Sophie is being treated under a continuum healthcare program; the Epilepsy Safety System (ESS) tele-monitors her health condition.

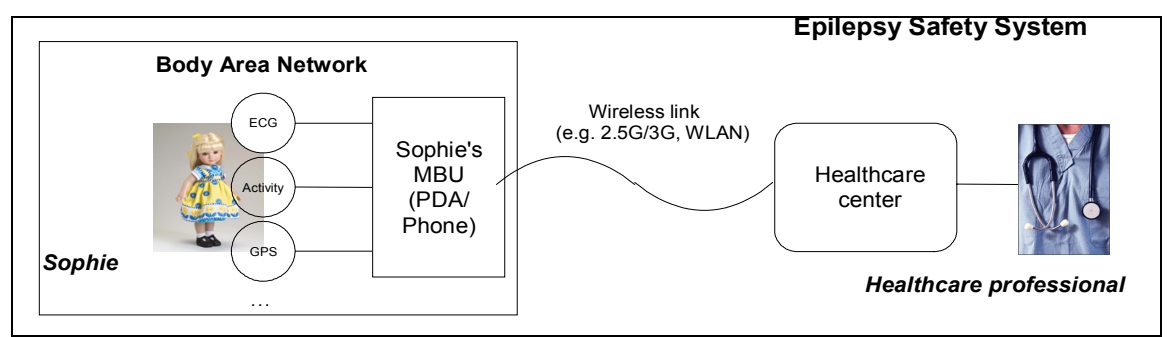

Fig.4. Epilepsy Safety System architecture

The ESS (Figure 4) is a distributed system, responsible for predicting, detecting and handling the occurrence of epileptic seizures. The ESS predicts if an epileptic seizure is likely to happen, based on Sophie's vital signs ${ }^{3}$. Therefore, she wears a Body Area Network (BAN), responsible for a collection of her vital signs data. The BAN consists of sensors (measuring ECG, activity), a GPS module (determining Sophie's location) and a Mobile Base Unit (MBU). A local ad-hoc communication network (e.g. Bluetooth) connects the sensors, GPS module and the MBU. The MBU is a gateway between the intra-BAN network and external $(2.5 \mathrm{G} / 3 \mathrm{G} / \mathrm{WLAN})$ communication networks and can be used to (locally) process the vital signs data. The MBU could be implemented as a Personal Digital Assistant (PDA) or a mobile phone. The BAN continuously monitors Sophie's vital signs, which are in real-time available to be viewed (e.g. on a PC) in the healthcare centre. This constitutes an m-health telemonitoring service.

To make Sophie's data available to her healthcare professional, the MBU connects to one of the external communication networks (e.g., WLAN, $2.5 \mathrm{G}$ or $3 \mathrm{G}$ ) as available at her current location and time. To support Sophie's mobility, the MBU supports seamless handover between these networks.

${ }^{3}$ According to the clinical research, an epilepsy seizure can be predicted at best 30 seconds before the seizure, based on ECG signals and an increasing heart rate of a patient. A prediction succeeds in $80 \%$ of the cases [8]. 
Sophie's doctor may always tele-monitor her vital sign's data in a reliable manner at the healthcare centre. This data is delivered within a maximum delay defined according to Sophie's current health condition: 1 second for an emergency (i.e. seizure) condition and 5 seconds otherwise. This delay is the time elapsed from the moment the data is gathered from Sophie's body to the moment it is displayed to her doctor in the healthcare centre. The doctor defined this delay requirement; such that in an emergency case, the healthcare centre can react in time and provide Sophie medical assistance.

Besides the delay, the doctor defined which basic data (e.g. alarm and location information in case of a seizure) must always be made available to him. He also defined which data is redundant (e.g. the full ECG signal).

The delay and basic/redundant data definitions constitute very important quality of service requirements for the tele-monitoring service. This is because the kind and volume of data transported to the healthcare centre depends on the capabilities of (i.e. quality of service offered by) communication networks available at Sophie's current location and time. Therefore, the MBU selects the external communication network depending on the quality of service offered by it.

Sophie works in a bank located in Paris city centre. In her workplace, there is WLAN available and whenever she leaves her job, there are $2.5 \mathrm{G} / 3 \mathrm{G}$ communication networks available in the city and its suburbs. At home, she also has a WLAN.

The ESS system has one more, very important feature. In case of a seizure, depending on its severity, the ESS alarms Sophie, the healthcare centre (i.e. her doctor) and eventually Sophie's mother (in case of a strong seizure). These activities take in total few seconds and they constitute an $\mathrm{m}$-health alarm service.

It is Saturday and Sophie is enjoying her day; she does not go to work. In the morning, she did some shopping in a local shopping mall. Now she wants to bike to the library and chooses a route via the city centre. Although Sophie feels good, her ESS warns her of a possible seizure and triggers a seizure alarm at the healthcare centre. She stops riding the bike and sits on a bench near-by. Before she can ask somebody for help, a seizure starts. ESS activates the m-health alarm service.

This time the seizure is very strong and ESS notifies her doctor and her mother.

The $3 \mathrm{G}$ network is available at Sophie's location. All Sophie's sensor data together with her location information is continuously sent to the healthcare centre. Sophie's doctor decides to intervene based on the ECG signals he sees on his screen. He sends an ambulance to the scene of the seizure.

Minutes later the ambulance reaches Sophie and medical professionals give her assistance and decide to take her to the hospital.

Sophie's doctor continues to monitor her while she is being transported. During the ride to the hospital, the ambulance moves out of the range of the $3 \mathrm{G}$ network and the MBU transparently switches to a $2.5 \mathrm{G}$ network. Once Sophie arrives at the hospital, the MBU connects to the available WLAN and her full ECG signals are automatically displayed in the emergency room.

After a final check-up and some rest, Sophie's mother meets her daughter in the hospital and takes her home.

When the MBU switches between different communication networks, the ESS adapts the signals it sends to Sophie's doctor. As result, the doctor will not see Sophie's vital 
signs when the ambulance moves out of the $3 \mathrm{G}$ network coverage into an are with only $2.5 \mathrm{G}$ coverage.

\subsection{Requirements for QoS- and context-aware mobile service infrastructure}

If we consider the QoS aspects of a context-aware service infrastructure like AWARENESS, we can conclude that we must meet with the following requirements to introduce QoS-awareness:

- QoS specification - The scenario shows that a service user, i.e., a doctor, is able to specify what health data must be always tele-monitored at the healthcare centre and what its delay upper-bound is. Concluding, the mobile service infrastructure must be able to handle user's end-to-end QoS specifications.

- QoS mapping - The end-to-end QoS specifications must be mapped into the QoS requirements of the underlying communication network infrastructure.

- Assessment of the QoS-context information - In case when multiple communication networks coexist, the mobile service infrastructure needs to select the most suitable one, depending on the QoS offered by it and QoS actually required.

- QoS adaptation - In case a communication network handover occurs, the QoS offered by the communication network infrastructure's changes and the service infrastructure should adapt (i.e. reduce data send or adapt an application protocol (section 5.5 provides details)).

- Context adaptation - In case of a context change, e.g., if an emergency occurs, the QoS requirements change. The service infrastructure should incorporate context information when mapping QoS and adapting to QoS changes.

- QoS monitoring - The service infrastructure must support the execution of real-time logging (i.e. measurements) of end-to-end QoS actually delivered to a mobile user.

\subsection{Requirements for the QoS-context source}

We indicate the QoS-context source as a source of the end-to-end QoS information aggregated over multiple mobile users. Following the classification given in [11] we distinguish technology (i.e. network) oriented and user-oriented end-to-end QoS characteristics that this source is going to aggregate.

Network-oriented end-to-end QoS characteristics:

- $\quad$ performance - expressed in speed ${ }^{4}$, accuracy ${ }^{5}$ and dependability ${ }^{6}$

\footnotetext{
4 Time interval used to transport data from a source to a destination [2].

5 The degree of correctness with which a service is performed [2].

6 The degree of certainty with which the service can be used regardless of speed or accuracy [2].
} 
User-oriented end-to-end QoS characteristics:

- perceived QoS - e.g. picture resolution, color accuracy, video rate and smoothness, audio quality, audio/video synchronization

- $\quad$ service cost - e.g. per use cost, per unit cost

- $\quad$ security level - e.g. authentication, confidentiality, integrity, non-repudiation

Requirements for a QoS-context source:

- $\quad$ dependability - source availability and reliability

- scalability - support for a number of users

- performance - real-time calculations and response to a mobile user

- QoS-context information management

$\circ$ aggregation, pre-processing, inference

- QoS prediction over different dimensions, e.g., prediction of QoS along the particular user trajectory in a particular timeframe

\subsection{Incorporating context}

To support our argument that the use of context in a mobile service infrastructure improves the delivered QoS, subsection 5.5.1 presents how application protocol adaptation can benefit from QoS-context information when transporting user data over the $3 \mathrm{G}$ wireless networks. Section 5.5.2 shows how location-specific QoScontext information can further improve QoS adaptation and delivered QoS.

\subsubsection{Application protocol adaptation}

To meet the required QoS, the mobile service infrastructure acquires from the QoScontext source information about the QoS offered by communication network infrastructures (WLAN/2.5G/3G) available at the user's current location and time.

One way of utilizing this information is adaptation of service delivery to the currently offered QoS. To illustrate it we use our knowledge about QoS offered by $3 \mathrm{G}$ communication network infrastructures. This comprises results of the performance evaluation of $3 \mathrm{G}$ communication networks as presented in $[12,13]$. Figure 5 shows the goodput ${ }^{7}$ and delay characteristics of a $3 \mathrm{G}$ network as a function of the application protocol's packet size sent in an uplink (i.e., from a mobile terminal to an application server). As can be expected a larger packet size results in a higher goodput, but is also accompanied with higher delays.

\footnotetext{
${ }^{7}$ Goodput - a throughput of a communication network infrastructure observed at the application layer [2].
} 


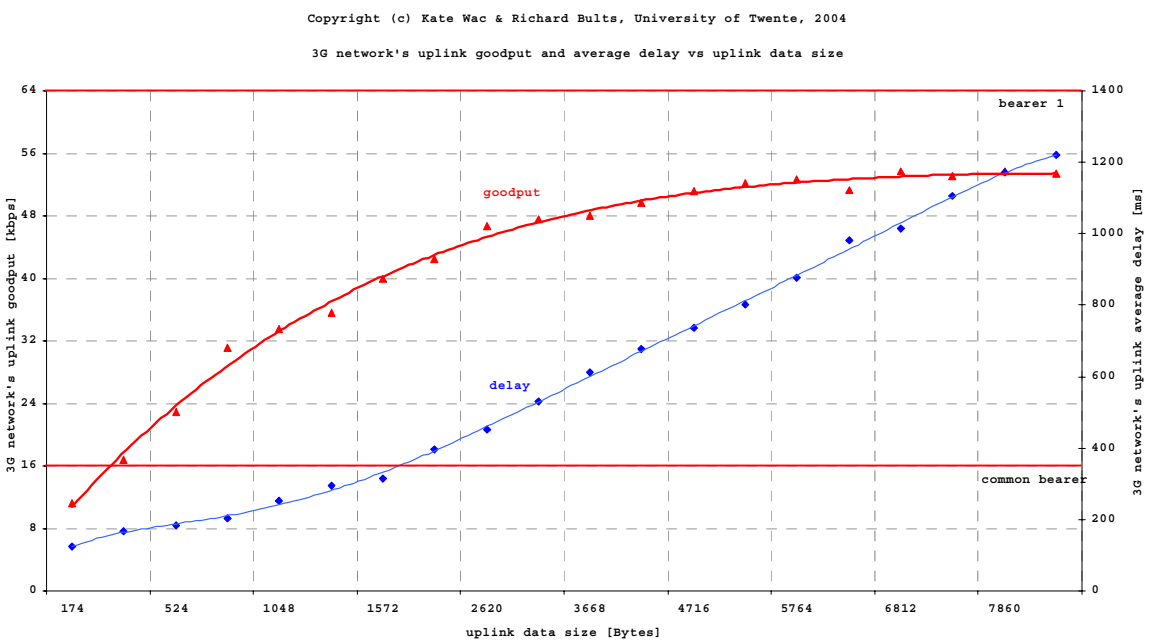

Fig.5. Performance characteristics of $3 \mathrm{G}$ communication network infrastructures

In a context-unaware situation, the application protocol designer maps a service user's QoS requirements to some fixed application protocol packet size; for a low-delay, a small packet size could be chosen and for an efficient (and cost-effective) use of the $3 \mathrm{G}$ network a larger packet size could be chosen. The choice for a packet size is a design-time choice.

If we incorporate context information in the application protocol, the packet-size could be adapted according to the context-dependent service user's QoS requirements. For Sophie's case that would mean that a large packet would be chosen in a noncritical situation and as soon as an emergency occurs (i.e., a seizure is predicted) the packet-size is reduced to the level that meets with the low delay QoS requirement.

\subsubsection{Location-and time-based QoS adaptation}

Another way of utilizing the QoS-context information is to conduct a seamless handover to the communication network offering better QoS than the network that is currently in use.

Because the service user is mobile, the underlying communication networks, and the QoS offered by these networks, change as the mobile user moves. The QoS offered by these networks depends, amongst others, on the user's actual location and time. It fluctuates over time because of a changing number of mobile users at a given location and their changing demands. Ideally, a QoS-context source provides this information into the service infrastructure in real-time. Furthermore, the QoS-context source needs to provide predictions of QoS along the particular user trajectory in a particular timeframe. This will guarantee that the mobile service infrastructure recognizes a QoS-context change and proactively acts upon that.

In a context-unaware situation, the system designer maps service user's QoS requirements to QoS offered by some default communication network. The choice for used communication network is a design-time choice. 
If we incorporate QoS-context information in the mobile service infrastructure, the choice of the actually used network is based on the context-dependent service user's QoS requirements.

For Sophie's case that would mean that if the seizure happens at $9.00 \mathrm{am}$, and her company WLAN offers a low QoS (due to people arriving at the office and downloading emails, documents etc), the system decides to use the $3 \mathrm{G}$ network to transmit her health signals and fulfill the tele-monitoring service's QoS requirements for an emergency.

\section{Conclusions}

In this paper, we discuss our ongoing research on QoS- and context-aware service infrastructure that supports the development of mobile applications in a heterogeneous network environment. We define a research problem and we indicate a possible research trajectory towards a validated solution. Hence, our research trajectory aims to prove that the use of context information plays a significant role in required QoS specification and it improves the delivered QoS. We indicate the necessity of a QoS-context source providing the service infrastructure with predictions on QoS offered by the networks at the service user's particular position and time. Due to the nature of the identified problem, we point prototyping as a preferred validation technique. Particularly we will validate if a mobile user experiences improvements in delivered QoS while using the QoS- and context-aware service infrastructure, comparing to the QoS-unaware one offering only the best-effort service.

One paper has been already published [14] disclosing our research topic and approving its relevance to the research community. We would like to indicate the importance of our eventual findings and its applicability to improve QoS delivered by any mobile service. The target audience of our research is any mobile service provider, and as currently more and more applications and services go mobile, we indicate a high need for our work. At the end the beneficiaries will be directly mobile users, experiencing their mobile services at the required quality. We would like to identify the newness of our approach expanding beyond the standard QoS management framework including QoS contracts and QoS negotiation components. We propose user-driven approach, where the user (and particularly a mobile application on user's behalf) will always have a choice between the underlying networks. The choice will be made with respect to user's QoS requirements.

Our research expands beyond the current telecom business model, where a user is locked to one mobile operator. In our view, the user needs to be able to make a decision, which network technology provided by which operator, is the most suitable to use. Moreover, by introducing the QoS-context source we further indicate usersempowerment; context information will be provided by users exclusively for users. 


\section{Acknowledgments}

I would like to gratefully thank Dr Aart van Halteren for his supervision. This work is a part of ongoing research in frame of the Freeband AWARENESS project. The Dutch government sponsors Freeband under contract BSIK 03025. This work is also partially supported by the E-NEXT Network of Excellence under contract FP6 IST 506869.

\section{References}

[1] D. Dey, Providing Architectural Support for Context-Aware applications, PhD thesis, Georgia Institute of Technology, USA, 2000

[2] ITU-T Recommendation I.350, General aspects of Quality of Service and Network Performance in Digital Networks, including ISDNs, March 1993

[3] Equanet project website, http://equanet.cs.utwente.nl

[4] CELLO project website, http://www.telecom.ntua.gr/cello

[5] J. Bolliger, T. Gross, A framework based approach to the development of network aware applications, IEEE Transactions on Software Engineering, vol. 24, no. 5, pp. 376 - 390, May 1998.

[6] D. Chalmers, M. Sloman, $Q o S$ and Context Awareness for Mobile Computing, Proc. 1st Intl. Symposium on Handheld and Ubiquitous Computing (HUC'99), Karlsruhe, Germany, pp. 380 -382, September 1999.

[7] J.-Z. Sun, J.Sauvola, J.Riekki, Application of Connectivity Information for Context Interpretation and Derivation, Proc. of 8th International Conference on Telecommunications (ConTEL 2005), 15-17 June 2005, Zagreb, Croatia

[8] M. Wegdam, AWARENESS: A project on Context AWARE mobile NEtworks and ServiceS, in 14th Mobile \& Wireless Communication Summit. 2005: Dresden, Germany

[9] I. Jacobson, G. Booch, J. Rumbaugh; The Unified Software Development Process; Addison-Wesley, 1999

[10]ITU-T Recommendation P.800, Methods for subjective determination of transmission quality, August 1998

[11]D. Chalmers, M. Sloman, A Survey of Quality of Service in Mobile Computing Environments, IEEE Communications Surveys, vol. 2, no. 2, 1999

[12]K. Wac, R. Bults, D. Konstantas, A. van Halteren, V. Nicola, Measurementsbased performance evaluation of $3 G$ wireless networks supporting $m$-health services, Proc. of Multimedia Computing and Networking 2005, IS\&T/SPIE Symposium on Electronic Imaging, 16-20.01.2005, CA USA

[13]R. Bults, K. Wac, A. van Halteren, V. Nicola, D. Konstantas, Goodput analysis of $3 G$ wireless networks supporting $m$-health services, Proc. of 8th International Conference on Telecommunications (ConTEL05), 15-17.06.2005, Croatia

$[14] \mathrm{K}$. Wac, A. van Halteren, T. Broens, Context-aware QoS provisioning in mhealth service platform, in 11th Open European Summer School (EUNICE 2005), 6-8 July 2005, Colmenarejo, Spain. 